\title{
On the Application of the Photoacoustic Methods for the Determination of Thermo-Optical Properties of Polymers
}

\author{
A. C. Bento*, D. T. Dias, L. Olenka, A. N. Medina, and M. L. Baesso \\ Universidade Estadual de Maringá, Departamento de Física, \\ Av. Colombo 5790, 87020-900, Maringá - PR, Brazil
}

Received on 6 November, 2001

\begin{abstract}
In this paper, the application of photoacoustic methods to study thermo-optical and spectroscopic properties of polymers is described. The Photoacoustic Spectroscopy, the Two-Beam Phase-Lag and also the so-called Open Photoacoustic Cell methods will be presented. The theoretical basis for quantitative measurements is discussed together with the advantages and limitations of the methods as compared with conventional measurements. Applications for spectroscopic and depth profile analysis and also for thermal properties measurements in several polymers samples are discussed.
\end{abstract}

\section{Introduction}

The sound effect induced in a heated solid by an intermittent light beam was first observed by Bell in the eighteenth century [1]. This effect was understood as a photothermal (PT) phenomena and was the origin of the so-called photoacoustic effect. Nowadays, there is a wide range of $\mathrm{PT}$ techniques and most of them were derived from the Photoacoustic Spectroscopy (PAS). Basically, their differences are related to the employed detection schemes. There are detection systems using: photodiodes [2], pyroelectrics [3, 4], piezoelectrics [5, 6], thermopiles [7], and microphones [8,9].

Photoacoustic (PA) is a photothermal phenomena that has been widely used to study the thermo-optical properties of materials. In brief, PA effect consists in illuminating a given sample with a modulated light beam and measuring the subsequent temperature fluctuation induced in the sample resulting from the light absorption, due to nonradiative de-excitation processes within the sample. Since the signal responds only to the absorbed light, the effects of scattered light play no significant role in these measurements. In addition, this method permit to solve the difficulties presented by the conventional Optical Spectroscopy [10-13], which does not generally allow studies of very weak absorbing materials and also opaque samples. Some PA studies in the infrared are based on Fourier Transform method (FTIR-PA), which allows the measurements to be performed in the medium infrared up to about $400 \mathrm{~cm}^{-1}$. PA is particularly useful in this region since it can detect the hydrogen bonds ( $\mathrm{C}-\mathrm{H}, \mathrm{O}-\mathrm{H}$ and $\mathrm{N}-\mathrm{H}$ ) that may present overtones and contribution from the combinations of the stretching and vibrational modes of the bonds. A review in the literature shows that a considerable number of the FTIR-PA study is in analytical sciences applied to polymer area [14-19].

In the polymer area, there are several kinds of non homogeneous samples that present high level of scattering light and also samples that are opaque in a considerable part of the spectrum. Due to that, photothermal method is recognized to be an important experimental procedure to access the thermo-optical properties of polymeric materials.

In this paper a selection of PA methods for characterization of polymers is presented. The UV-VIS Photoacoustic Spectroscopy (PAS) and Phase Resolved PAS, the Two-Beam Phase-Lag and also the so-called Open Photoacoustic Cell (OPC) [13] will be described The theoretical basis and experimental results together with the perspectives of future studies in this area will also be discussed.

\section{Standard Rosencwaig-Gersho model modified for two-beam accessment}

The PA effect can be produced by any kind of absorption that results in a periodical heating. Some schemes of PA generation are depicted in Fig. 1. Although the heat diffusion model (Thermal Piston) [8] is the most common mechanism responsible for the generation of the PA signal, effects like surface displacement (Thermal Expansion) [20], thermoelastic bending (Drum Ef- 
fect) $[21,22]$ and the gas evolution (Photobaric Effect) [23] may also contribute to produce a detectable acoustic signal. All these effects result in a pressure variation inside the gas chamber of the photoacoustic cell.

PHOTOACOUSTIC GENERATION SCHEME

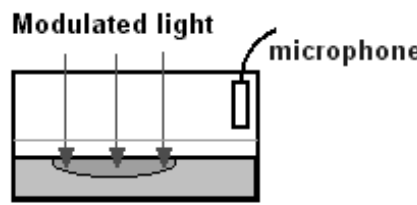

Thermal expansion $\delta \mathrm{L} \Rightarrow>$ Pac

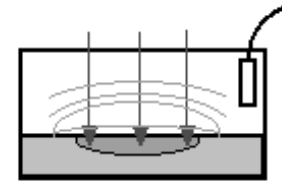

Thermal Diffusion $\delta \mathrm{T} \Rightarrow$ Pth

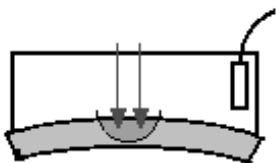

Thermoelastic bending $\delta u_{Z}+\delta u_{I} \Rightarrow P$ Pac

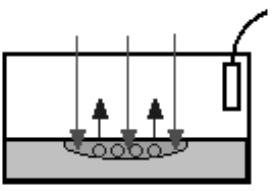

Photobaric effect $\mathrm{h} v==>\delta \mathrm{Pac}$

Figure 1. Possible PA generating mechanisms.

The Rosencwaig-Gersho(RG) theoretical treatment for the photoacoustic effect (known as thermal piston model) considers that the pressure inside the photoacoustic cell is proportional to heat generated by the absorbed light and depends on both the geometry of the cell and the thermo-optical properties of the investigated sample. The two-beam modified RG model [21] can be established using the diagram shown in Fig. 2 . The one-dimensional coupled differential equations that describe the heat diffusion are given as follow [24]

MODIFIED ROSENCWAIG\&GERSHO THERMAL DIFFUSION MODEL

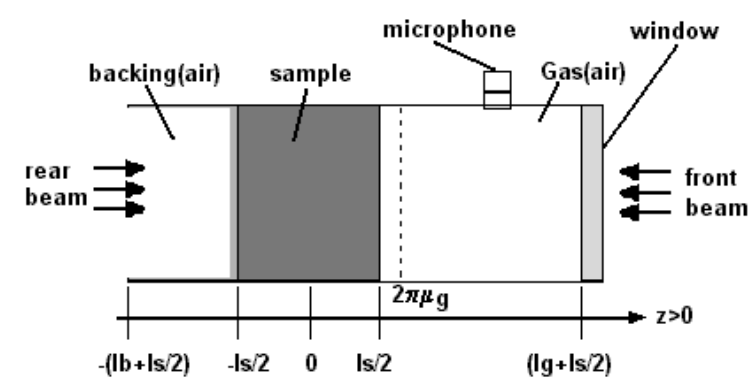

Figure 2. RG based PA geometry for dual beam.

$$
\frac{\partial^{2}}{\partial z^{2}} \theta_{i}(z, t)-\frac{1}{\alpha_{i}} \frac{\partial}{\partial t} \theta_{i}(z, t)=-F_{i}(z, t)
$$

with $i=" g "($ gas $)=>\left(l_{g}+l_{s} / 2\right)<z<l_{s} / 2$; $i=$ " $b$ " (backing) $=>-l_{s} / 2<z<\left(-l_{s} / 2+l_{b}\right)$; $i=" s "($ sample $)=>l_{s} / 2<z<\left(-l_{s} / 2\right)$; the term $F_{i}(z, t)=\left(f_{i}(z, t) / k_{i}\right)$ represents the heat source, being $f_{g}(z, t)=f_{b}(z, t)=0$ and $f_{s}(z, t) \neq 0 ; \alpha_{i}$ is the thermal diffusivity, defined as $\alpha_{i}=k_{i} /\left(\rho C_{p}\right)_{i} ; k_{s}$ is the thermal conductivity, $\rho C_{p}$ is the volumetric heat capacity, and $l_{i}$ is a length. Boundary conditions for the continuity of temperature and heat flow are specified for adjacent mediums at the interface $i j$ as

$$
\theta_{i}=\theta_{j}, \quad k_{i} \frac{\partial}{\partial z} \theta_{i}=k_{j} \frac{\partial}{\partial z} \theta_{j}
$$

The solution of coupled equations is

$$
\theta\left(l_{s} / 2, t\right)=\frac{-1}{k_{s} \sigma_{s}} \int_{-l_{s} / 2}^{l_{s} / 2}\left[\frac{(b-1) e^{-\sigma_{s}\left(z+\frac{l_{s}}{2}\right)}-(b+1) e^{\sigma_{s}\left(z+\frac{l_{s}}{2}\right)}}{(g-1)(b-1) e^{-\sigma_{s} l_{s}}-(g+1)(b+1) e^{\sigma_{s} l_{s}}}\right] f(z, t) d z,
$$

in which

$$
b=\frac{k_{b} a_{b}}{k_{s} a_{s}}, \quad g=\frac{k_{g} a_{g}}{k_{s} a_{s}}, \quad r=(1+j) \frac{\beta}{2 a_{s}}=\frac{\beta}{\sigma_{s}} .
$$

The complex parameter $\sigma_{i}=(1+j) a_{i}$ is frequency dependent, in which $a_{i}=\left(\omega / 2 \alpha_{i}\right)^{1 / 2}$ and $\mu_{i}=1 / a_{i}$ named as the thermal diffusion length; $\beta$ and $l_{\beta}=1 / \beta$ are the absorption coefficient and optical absorption length, respectively.

\section{Front illumination}

Using the illumination $I(t)=I_{o}\left(1+e^{j \omega t}\right)$, the density of absorbed power can be expressed as $f(z, t)=$ $-\beta I_{o} e^{-\beta\left(l_{s} / 2-z\right)}\left(1+e^{j \omega t}\right)$. Solving the integral in Eq. (3) we have the thermal fluctuation on sample surface as: 


$$
\theta_{F}\left(l_{s} / 2\right)=\frac{\beta I_{o}}{k_{s} \sigma_{s}^{2}\left(r^{2}-1\right)}\left[\frac{(b+1)(r-1) e^{\sigma_{s} l_{s}}-(b-1)(r+1) e^{-\sigma_{s} l_{s}}+2(b-r) e^{-\beta l_{s}}}{(g+1)(b+1) e^{\sigma_{s} l_{s}}-(g-1)(b-1) e^{-\sigma_{s} l_{s}}}\right] .
$$

this is the RG derived equation. This temperature is damped in the gas length $2 \pi \mu_{g}$ and the acoustic signal is given by

$$
S_{F}=\left|\frac{\gamma P_{o} \theta\left(l_{s} / 2\right)}{l_{g} \sigma_{g} T_{o}}\right| e^{j \Phi_{F}} .
$$

The gas filling the PA cell is assumed to be an ideal gas with $P V^{\gamma}=$ cte, with $\gamma=C_{p} / C_{v}$ as the gas spe- cific heat ratio; $P_{0}$ and $T_{0}$ are the ambient pressure and temperature. The Eq. (6) gives a complex number that has a module $\left\|S_{0}\right\|$ and a phase $\Phi_{0}$.

\section{Rear illumination}

In this geometry, we assume $I(z)=I_{o}(1+$ $\left.e^{-\beta\left(l_{s} / 2+z\right)}\right)$ and the heat source in the form $f(z)=$ $\beta I_{o} e^{-\beta\left(l_{s} / 2+z\right)}$, thus Eq. (3) gives

$$
\theta_{R}\left(l_{s} / 2\right)=\frac{\beta I_{o}}{k_{s} \sigma_{s}\left(r^{2}-1\right)} \frac{\left[(b+1)(r+1) e^{\sigma_{s} l_{s}}-(b-1)(r-1) e^{-\sigma_{s} l_{s}}\right] e^{-\beta l_{s}}-2(b+r)}{(g+1)(b+1) e^{\sigma_{s} l_{s}}-(g-1)(b-1) e^{-\sigma_{s} l_{s}}} .
$$

By taking the relation of $\mu_{s}$ with $l_{s}$ and $l_{\beta}$, the expressions (5) and (7) can be simplified since one considers some limiting cases in the PA model. They take into account the thermal thickness (thermally thick $\left(\mu_{s}<<l_{s}\right)$ or thermally thin $\left.\left(\mu_{s}>>l_{s}\right)\right)$ and the opacity (transparent $\left(l_{\beta}>>l_{s}\right)$ and opaque $\left.\left(l_{\beta}<<l_{s}\right)\right)$. In the case of spectroscopic study, highly absorbing sample may present a saturated PA spectrum $\left(\beta l_{s}>>1\right)$, which constitutes in a serious trouble for resolving the optical absorption bands. Fortunately, as opacity depends on thickness, usually one can drive the PA spectrum to a resolved case $\left(\beta \mu_{s}<1\right.$ and $\left.\beta l_{s}<1\right)$, by adjusting sample thickness or selecting a suitable modulation frequency range.

As mentioned before, for polymer samples, PAS is particularly useful in the near infrared (NIR) and medium infrared (MIR), where Hydrogen bonds (C-H, $\mathrm{O}-\mathrm{H}$ and $\mathrm{N}-\mathrm{H}$ ) may present overtones and contribution from the combinations of the stretching and vibrational modes.

\section{Open Photoacoustic Cell}

The Open PA Cell consists in a commercial electret microphone that receives directly on top a flat slab of a solid sample [25, 26]. The microphone is located in the backing position if we consider the geometry of Fig. 2. A metallized Teflon foil $12 \mu \mathrm{m}$ thick with a coating film of $500-1000 \AA$ transduces the pressure inside a minimum volume chamber with an air gap $\sim 45 \mu \mathrm{m}$. Pressure variation induces a voltage in the electret and for an opaque sample it follows that

$$
P=\frac{\gamma P_{0} I_{0}\left(\alpha_{g} \alpha_{s}\right)^{1 / 2}}{2 \pi l_{g} T_{0} k_{s} f} \frac{e^{j\left(\omega t-\frac{\pi}{2}\right)}}{\sinh \left(\sigma_{s} l_{s}\right)} .
$$

The limiting cases are given for two situations: thermally thin $l_{s} a_{s} \ll 1$, where pressure is dependent on $f^{-3 / 2}$, and for thermally thick $l_{s} a_{s} \gg 1$, with signal decaying exponentially with frequency as $S \propto(1 / f) . e^{-b \sqrt{f}}$. Fitting data to have $b$, thermal diffusivity is obtained from $\alpha_{s}=\left(\pi l_{s}^{2} / b^{2}\right)$. Even though this method is designed for opaque sample, it is accepted that a thermally thin evaporated coating on a transparent sample or a very thin metal foil fixed on it can also be studied with this procedure[27].

\section{Photoacoustic methods}

\section{IV.1 Depth profiling: frequency scanning}

If the light is absorbed in a length $l_{\beta}$, the only detectable heat is that inside the thermal diffusion length $\mu=(\alpha / \pi f)^{1 / 2}$, which gives the "thermal skin depth" of the sample. Varying frequency $f$, one can perform the depth profiling of a layered sample, Fig. 3. The parameter $\mu$ classifies sample in two category is thermally thick $\left(\mu_{s}<<l_{s}\right)$ or thermally thin $\left(\mu_{s}>>l_{s}\right)$ and for $\mu_{s}=l_{s}$ we define the critical frequency $f_{c}=\left(\alpha / \pi l^{2}\right)$. This method is applied for both spectroscopic analysis 
(varying wavelength) and thermal properties measurements (fixed wavelength and varying frequency).

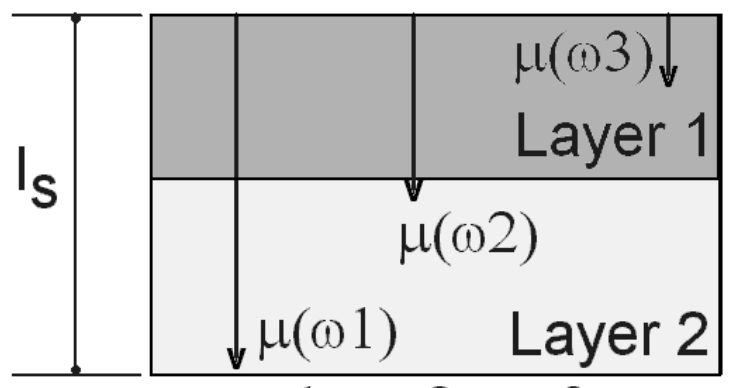

$\omega 1<\omega 2<\omega 3$

Figure 3. Depth penetration $\mu(\omega)$ for a layered sample.

\section{IV.2 Phase Resolved method}

Phase Resolved is a numerical method used in PAS [28] to separate a spectrum, when it is composed by more than one absorbing component. For example, PA signals $S_{A}\left(\lambda_{1}\right)$ and $S_{B}\left(\lambda_{2}\right)$ with bands superimposed and being centered at two near wavelengths $\left(\lambda_{1} \sim \lambda_{2}\right)$. In principle, if PA signals present a phase difference $\Delta \phi \neq 0$ at a chosen wavelength we may be able to resolve the spectra for each component. The instrumental accuracy requires $\Delta \phi$ greater than $5^{0}$. In this method, PA signal is represented in a phasorial picture, where it should be separated in two components: in phase and in quadrature as shown in Fig. 4. For a particular projecting angle $\Theta_{B}$ we have separated contribution from absorbing center $B$ and its PA signal is maximum at $\Theta_{B} \pm 90^{\circ}$.
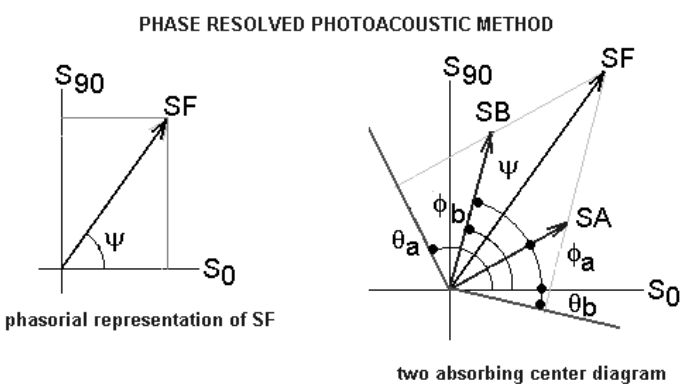

$S_{\theta}(\lambda)=S_{0}(\lambda) \cos (\theta)+S_{90}(\lambda) \operatorname{sen}(\theta)$

$$
\psi=\phi_{\mathrm{b}}-\phi_{\mathrm{a}}
$$

projecting $S F$ at $\theta_{b}$ separates $S_{B}$ and $S_{B} \max$ is at $\left(\theta_{b} \pm 90\right)$

projecting $S F$ at $\theta a$ separates $S_{A}$ and $S_{A}$ max is at $(\theta a \pm 90)$

Figure 4. Phasorial diagram for Phase Resolved PAS.

\section{IV.3 Thermal expansion}

For a uniform heating, there is no temperature gradient on sample (see Fig. 1), thus thermal expansion may be present. Solving the coupled differential equations by considering sample poor thermal conducting $(b>r)$, transparent $\left(l_{\beta}>l_{s}\right)$ and in the thermally thick $\left(\mu_{s}<l_{s}\right)$, the surface displacement $\Delta z=l_{s} \alpha_{T} \theta_{s}$ is evaluated. If no heat is transferred to the gas $\left(k_{s} \frac{\partial \theta_{s}(0)}{\partial z}=0\right.$, sample-gas interface), then the pressure is $\delta P=\left(\gamma P_{o} l_{s} / l_{g}\right) \alpha_{T} \theta_{s} e^{j \omega t} \quad$ [29] and the temperature

$$
\theta_{s} \simeq \frac{-\beta I_{0}}{\sigma_{s}^{2} k_{s}\left(\frac{\beta}{\sigma_{s}}-1\right)} \frac{1}{(1+j \omega \tau)} .
$$

By taking the module and the phase of Eq. (9) both the thermal diffusion time $\tau$ and the non-radiative relaxation time $\tau_{\beta}=\left(1 / a_{s} \beta^{2}\right)$ are calculated.

\section{IV.4 Thermoelastic bending}

When a flat sample is under a non-uniform heating, expansion and contraction may undergo to a thermal gradient (see Fig. 1), thus a thermoelastic bending is likely to rise as displacement $u_{z}\left(r, l_{s} / 2\right)[21,22]$. This effect takes into account the sample's displacement in both direction $u_{r}$ radial and $u_{z}$ longitudinal or $z$ (normal to sample plane). The temperature $\theta_{s}$ is calculated from Eq. (3) by considering strong surface absorption and using $f\left(z_{0}\right)=-\beta_{s} I_{0} \delta\left(z_{0}\right)$. It follows that

$$
\begin{gathered}
\theta_{s}\left(l_{s} / 2\right)=\frac{\beta_{s} I_{o}}{k_{s} \sigma_{s}} \frac{\cosh \left[\sigma_{s}\left(z \pm \frac{l_{s}}{2}\right)\right]}{\operatorname{senh}\left(\sigma_{s} l_{s}\right)}, \\
P_{t h}=\frac{\gamma P_{o}}{T_{o} l_{g}} \int_{o}^{2 \pi \mu_{g}} \theta_{s}\left(l_{s} / 2\right) e^{-\sigma_{s} z} d z,
\end{gathered}
$$

$\theta_{s}$ is the thermal contribution and $\beta_{s}$ is a dimensionless absorption coefficient and the signal " + " means front $\left(\theta_{F}\right)$ and "-" is for rear $\left(\theta_{R}\right)$.

Integrating the displacement produced by the front illumination the acoustic pressure is obtained [22] $P_{a c}=$ $\gamma P_{o} / V_{o} \int_{o}^{R^{\prime}} 2 \pi \mu_{g} u_{z}\left(r, l_{s} / 2\right) d r$. According to McDonald and Wetzel [30], the total pressure is then $P=$ $P_{t h}+P_{a c}$. If thermoelastic bending is present, one can retrieve thermal properties from the phase of the photoacoustic signal. In the thermally thick case (high $f$ with $l_{s} a_{s} \gg 1$ ), the phase of the signal as a function of the modulation frequency has the form:

$$
\phi_{\mp}=\mp \frac{\pi}{2}+\arctan \left[\frac{1}{Z-1}\right]
$$

where "- " refers to $\phi_{F}$ and "+ " to $\phi_{R}$, yet $Z=l_{s} a_{s}=b \sqrt{f}$ is a parameter to be fitted that 
allows one to calculate thermal diffusivity $\alpha_{s}$ using $b=\left(\pi l_{s}^{2} / \alpha_{s}\right)^{1 / 2}$.

\section{IV.5 Two-Beam phase lag method}

The Two-Beam Geometry uses the signals $S_{F}$ (front beam) and $S_{R}$ (rear beam), which are obtained from the modified RG model (see Fig. 2) [31]. It requires an air backing $(g=b=0)$, opaque condition $\left(\beta l_{s} \gg 1\right)$ and sample must have surface absorption $\left(\beta \gg a_{s}\right)$. The typical two beam PA signals ratio and the phase lag are given by $\left(S_{F} / S_{R}\right)=\left(I_{F} \beta_{F} / I_{R} \beta_{R}\right)\left[\cosh ^{2}(Z)-\right.$ $\left.\sin ^{2}(Z)\right]^{1 / 2}$ and $\tan (\Delta \phi)=\tanh (Z) \cdot \tan (Z)$, with $Z=$ $l_{s} . a_{s}$ and $\Delta \phi=\left(\phi_{F}-\phi_{R}\right)$. One can evaluate the parameter $Z$ from $\tan (\Delta \phi)$ at a single frequency. Thus, thermal diffusivity $\alpha_{s}$ is obtained using $\alpha_{s}=\pi f\left(l_{s} / Z\right)^{2}$. This model was adapted for transparent samples by forcing optical absorption coefficient at the sample surface, e.g., using a metallic coating or a thin Al foil [27].

\section{IV.6 Photoacoustic typical arrangements}

Figure 5 shows the experimental setup for PAS, a homemade PA spectrometer. In this setup, white light (Halogen or Xe arc lamp, $150 \mathrm{~W}$ to $1000 \mathrm{~W}$ ) passes throughout a monochromator (180 nm to $3800 \mathrm{~nm})$. A mechanical chopper modulates the light and the beam goes to the PA cell. PA conventional cell must have an optical window to allow radiation to reach the testing sample placed inside. A chopper driver $(1 \mathrm{~Hz}$ to 3 $\mathrm{kHz}$ ) gives the pulse reference that synchronizes the PA signal $(1 \mu \mathrm{V}$ to $2 \mathrm{mV}$ ) collected by the microphone (20 $\mathrm{mV} / \mathrm{Pa}$ to $50 \mathrm{mV} / \mathrm{Pa}$ ), which is monitored by a Lock-in amplifier. The PA experiment runs varying two main variables: wavelength or frequency. Alternatively, OPC uses the same principle but, for the thermal property evaluation, sample is placed on top of a commercial electret's microphone. The sample consists itself in a sealing for the PA cell. For the OPC, usually it is used a fixed wavelength (UV, VIS or IR), a laser beam or a white light.

\section{EXPERIMENTAL SETUP FOR PHOTOACOUSTIC SPECTROSCOPY}

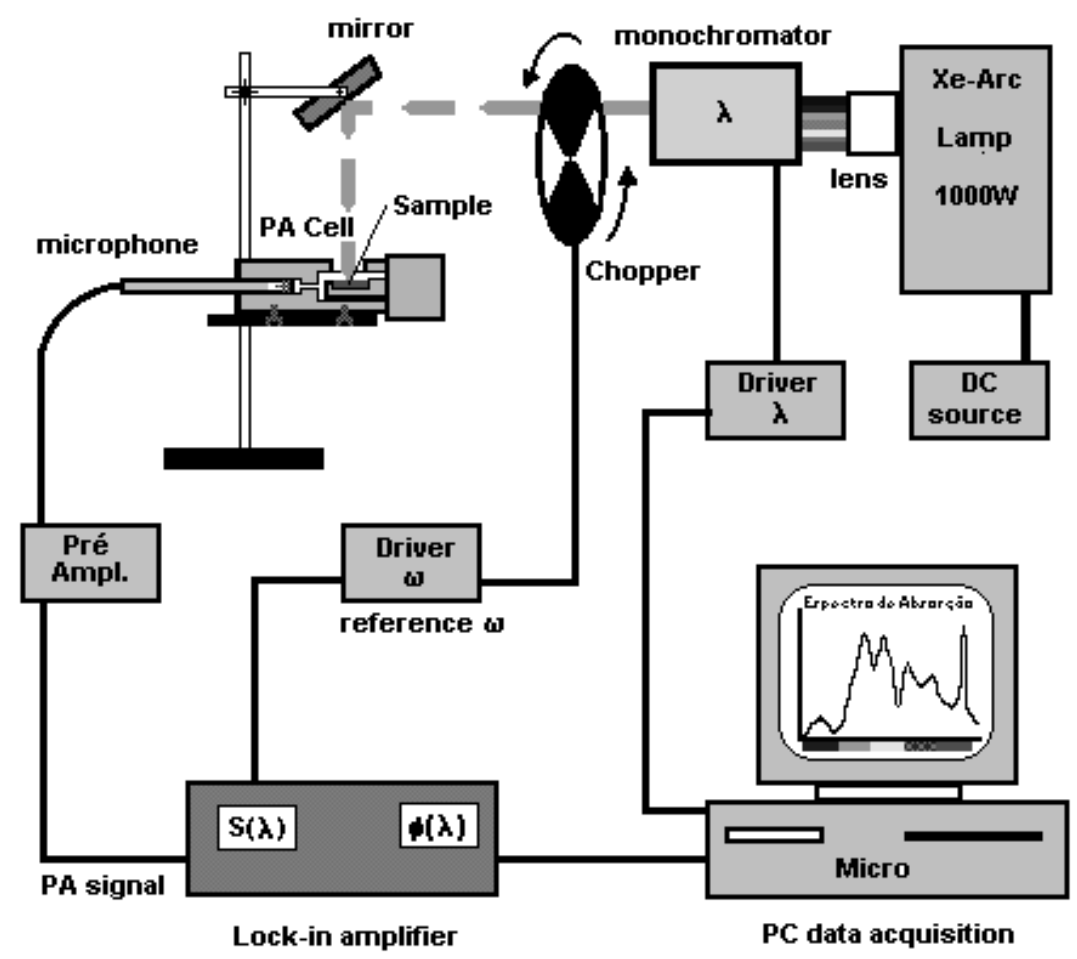

Figure 5. Conventional PAS setup. 


\section{Photoacoustic spectroscopy applied to polymers}

\section{V.1 Infrared PA depth profiling}

Many papers are found in literature about polymer's study using FTIR with PA detection. Few of them are about polymerization of lactones under Hydroxyapatite presence and in Hydroxyalkyl Methacrylate, water soluble Acryllamide copolymer, structural transformation of $\mathrm{PE}$ phase in blends of oriented PE-Polypropylene (PEPP), interfacial ties in thermoplastics olefins during stratification [14, 16, 17, 32, 33], whilst many others treat the depth profiling analysis in analytical science [18]. Studies on compositional gradients in urethanes and latexes, chain scission in UV exposed epoxy and urethane films, individual distribution of thermoplastic through stratification near surface in PP and EthylenePropylene rubber, photo oxidation in Styrene-Isoprene copolymer are also reported. [19, 34, 35, 36, 37].

The ability of the FTIR-PAS for depth profiling analysis in layered materials has been demonstrated by Dittmar and coworkers [38], that use the thermal diffusion length $\mu=(\alpha / \pi \omega)^{1 / 2}$ as a key parameter. They discussed about the dependence of the PA signal and thermal diffusion length in homogeneous and inhomogeneous laminar samples. This technique was applied on a Vinyl resin (Ethylene Vinyl Acetate-EVA, DuPont Elvax) that was cast onto a PP substrate, and to study a Polyamide film (DuPont Kapton) that was sandwiched between two layers of Fluorethylene (Teflon). From phase resolved analysis they separated the surface and bulk contribution for the spectra of Polyamide. The study of the integrated intensities of the EVA-on-PP showed to be useful in locating the layers where the band contributions come from. They located the carbonyl stretch at $1730 \mathrm{~cm}^{-1}$ (surface layer), the $\mathrm{C}-\mathrm{H}$ bends at $1370 \mathrm{~cm}^{-1}$ and at $1450 \mathrm{~cm}^{-1}$ (surface and bulk), C-C lattice vibration at $1160 \mathrm{~cm}^{-1}$ (bulk layer), the $\mathrm{C}-\mathrm{H}$ stretching peaks at $2918 \mathrm{~cm}^{-1}$ and at 2850 $\mathrm{cm}^{-1}$.

A similar depth analysis in near infrared (NIR) in the range between $1.0 \mu \mathrm{m}$ up to $3.0 \mu \mathrm{m}$ is presented by Oliveira et al. [39]. Scanning a PE slab of $1 \mathrm{~mm}$ thick using frequency in the range of $10 \mathrm{~Hz}$ to $240 \mathrm{~Hz}$ they probed depth in the range between about $56 \mu \mathrm{m}$ and $11 \mu \mathrm{m}$. The aim was to study the location of $-\mathrm{CH}_{3},=\mathrm{CH}_{2}$ and $-\mathrm{OH}$ groups. They studied the Low-Density PE (LDPE) through PA peak intensities ratios of $-\mathrm{CH}_{3},=\mathrm{CH}_{2}$ and $-\mathrm{OH}$ groups related to that of methylene group. They observed that these ratios increased, showing that the layers of $\mathrm{PE}$ closer to surface are richer in $-\mathrm{CH}_{3},=\mathrm{CH}_{2}$ and $-\mathrm{OH}$ groups as compared to the bulk.

\section{V.2 Visible optical absorption of polyethylene}

A useful method have been presented by Vargas et al. [40] for optical absorption measurements in a new composite material based on $\mathrm{PE}$ coated. It combines PA and transmission techniques. The method states that a solid sample of thickness $l$, reflectivity $R(\lambda)$ and absorption coefficient $\beta(\lambda)$, will present a transmitted beam intensity $T(\lambda)$ and the PA signal $S(\lambda)$. It follows that $T$ and $S$ can be represented explicitly by terms dependent of $\lambda$, i.e., $T(\lambda)=(a / b) \exp (-\beta(\lambda) . l)$ and $S(\lambda)=a[1-\exp (-\beta(\lambda) . l)]$. Parameters $a$ and $b$ depend on $\lambda$ through $R(\lambda)$. Four testing samples were used and the layer of $\mathrm{MnO}_{2}$ presented thicknesses in the range of $17 \mathrm{~nm}$ to $200 \mathrm{~nm}$. The PA spectra and transmission data were obtained from 450 $\mathrm{nm}$ up to $650 \mathrm{~nm}$, being $M n$ peaking at $450 \mathrm{~nm}$. The authors have shown that for calculating $\beta(\lambda)$ one have to invert the $T(\lambda)$ and $S(\lambda)$ equation and obtain specifically an expression to give $\beta$ independently as $\beta_{T}(\lambda)=(1 / l) \ln \{a(\lambda) /[T(\lambda) \cdot b(\lambda)]\}$ and $\beta_{S}(\lambda)=$ $(1 / l) \ln \{1-S(\lambda) / a(\lambda)\}^{-1}$. They derived $a(\lambda)$ and $b(\lambda)$ by fitting the plots of $S(\lambda)$ versus $T(\lambda)$ which is linear. The values found showed an exponentially decaying curve of $\beta_{\text {average }}(\lambda)$ that ranges from $1.0 \mathrm{~cm}^{-1}$ to $3.210^{5} \mathrm{~cm}^{-1}$ when $\lambda$ goes from $450 \mathrm{~nm}$ to $650 \mathrm{~nm}$. The work also pointed out that such a high optical absorption coefficient could be useful in the lining solar collectors.

\section{V.3 Thermal diffusion and non-radiative relax- ation time}

Another PA application is the investigation of the kinetics of the iodine doping process [41]. Iodine Doped Polystyrene (PST: $I_{2}$ ) was prepared using an atactic PST film that were cast from $5 \%(\mathrm{w} / \mathrm{w})$ chloroform solution over a flat clean glass plate, exposing PST films to vapor phase iodine. PAS spectrum of the PST: $I_{2}$ showed two main peaks at $310 \mathrm{~nm}$ and $495 \mathrm{~nm}$. The RG model is modified for a thermally thick sample, by including the effects of a finite non-radiative de-excitation time. The dependence of the phase in thermally thick regime is rewritten similarly to that presented for thermal expansion and suitable for transparent materials. The phase is given by

$$
\Phi(\omega)=-\frac{3 \pi}{4}-\tan ^{-1}(\omega \tau)+\tan ^{-1}\left[\frac{1}{\sqrt{2 \omega \tau_{\beta}}+1}\right]
$$

showing that phase depends on both $\tau$ and $\tau_{\beta}$. Results showed $\tau_{310}$ greater than $\tau_{495}$ but both presenting a minimum $\tau \sim 20 \mathrm{~ms}$ for $t=120 \mathrm{~min}$ and $180 \mathrm{~min}$. In contrast, it was observed $\tau_{\beta 310}$ smaller than $\tau_{\beta 495}$ but peaking to $\tau_{\beta} \sim 100 \mathrm{~ms}$ in the same interval of time. This behavior for $\tau$ and $\tau_{\beta}$ was explained as a second-order phase transition.

\section{V.4 Dyeing monitoring in PET films}

Generally, PE-Terephthalate (PET) fiber that is applied mainly with cotton in textile industries should 
be adequately dyed in order to present a better appearance [42]. The PAS technique is applied to N,N-Dimethylacrylamide (DMMA) modified and nonmodified PET films that have been dyed with Samaron Blue HGS dye and with Dianix FB-E Red dye. Mainly, visible optical absorption bands of Blue Dye at $632 \mathrm{~nm}$ and bands of Red Dye at $442 \mathrm{~nm}$ were monitored using the PAS spectra as a function of processing parameter. It was found that the best condition for dyeing is a $2 \%$ bath concentration, 15 min for films treatment at $85^{\circ} \mathrm{C}$ with the modifier DMMA and after that, dyeing over $30 \mathrm{~min}$ at $85^{\circ} \mathrm{C}$. No difference in processing method was found when both disperse dyes are compared.

\section{V.5 Cross-linking process}

Here it is shown the possibility of using the PAS to evaluate the cross-linking of the copolymer from EVTrimethoxysilane (EVS), named Cop, and the grafted Vinyl Trimethoxysilane (VTS) on LDPE, named PEg. PAS is used for monitoring the overtone bands and stretching frequencies combinations of the groupings $-\mathrm{Si}-\mathrm{OH},=\mathrm{CH}_{2},-\mathrm{CH}_{3}$ and $-\mathrm{CH}_{2}-\mathrm{CH}_{3}$, in the near and medium infrared range. Throughout PAS spectra, using the assigned absorption bands [39], the comparison between a reference and a cross-linked sample is possible. PAS is used to study peaks evolution in both, copolymer EVS and grafted LDPE. The samples were typically prepared with $3 \%, 5 \%$ and $7 \%$ of catalyst and cross-linked at 70, 80 and $90{ }^{\circ} \mathrm{C}$, respectively, which resulted in nine samples. The typical infrared PA spectrum is shown in Fig. 6 and the best combinations of the peak ratios are depicted in Fig. 7 for NIR. Table I presents the tabulated bands assignments for PE.

Taking the analysis of the overtone bands of $-\mathrm{OH}$ groups, the better cross-linked sample was found to be that prepared with $80{ }^{\circ} \mathrm{C}$ and in the range of $5 \%$ to $7 \%$ of catalyst, typically, as one can observe by looking at the central frames of Fig. 7 [43].

Phase Resolved PA method may be used in spectroscopy for separating spectra in sample with more than one absorbing center at a wavelength in both range NIR and MIR [38]. In principle, if there is phase difference at a sorted PAS peak we may be able to resolve the spectra for each component, regarding instrumental accuracy $\sim 5^{0}$. The PA spectra for Cop and PEg and their phases showed a phase difference $\sim 7^{0}$ for Cop and $\sim 10^{\circ}$ for the superimposed peaks 2 and 3 , in the NIR region. The phase separation for PEg770 and Cop770 has shown that peak 2 is separated at a phase of $50^{\circ}$, which means that its maximum is at $140^{\circ}$. On the other hand, peak 3 is separated at a phase $35^{\circ}$ with maximum at $125^{\circ}$. In the same way the peak 2 and peak 3 of Cop770 is also separated.

Table I - Tabulated infrared bands assigned to PE in NIR and MIR range.[39]

\begin{tabular}{|c|c|c|}
\hline $\begin{array}{l}\text { Wavelength } \\
\mathrm{nm}\left\{\mathrm{cm}^{-1}\right\}\end{array}$ & $\begin{array}{c}\text { Observed } \\
\text { Peaks }\end{array}$ & $\begin{array}{l}\text { Assignment } \\
\text { [observation] }\end{array}$ \\
\hline $1250\{8000\}$ & 1 & 2nd overtone [characteristic of $-\mathrm{CH}_{2-}$ and $\mathrm{CH}_{3^{-}}$groups] \\
\hline $1340\{7460\}$ & - & $\begin{array}{l}\text { Probably a combination [characteristic of terminal olefin methylene } \\
\text { group] }\end{array}$ \\
\hline $1400\{7142\}$ & 2 & Free $O H, 1$ st overtone \\
\hline $1420\{7042\}$ & 3 & Combination $\nu_{C H}{ }^{*}+\left[-\mathrm{CH}_{2}-, \mathrm{CH}_{3}-\right.$ groups $]$ \\
\hline $\begin{array}{l}1450-1490\{6896- \\
6711\}\end{array}$ & - & Bound $\mathrm{OH}, 1$ st overtone[superimposed to $\nu_{C O}, 3$ rd overtone] \\
\hline $1760\{5681\}$ & 4 & 1st overtone [characteristic of $-\mathrm{CH}_{2}-$ groups] \\
\hline $\begin{array}{l}1800-1920\{5555- \\
5208\}\end{array}$ & 5 & Free $O H$, combination \\
\hline $2020\{4950\}$ & 6 & $\begin{array}{l}\text { Probably a combination [characteristic of terminal olefin methylene } \\
\text { group] }\end{array}$ \\
\hline $\begin{array}{l}2080-2140\{4807- \\
4672\}\end{array}$ & 6 & $\begin{array}{l}\text { Combination } \nu_{C H}{ }^{*}+[\text { characteristic of terminal olefin methylene group } \\
\mathrm{O}-\mathrm{CH}=\mathrm{CH}_{2} \text {. Region of } \nu_{\mathrm{OH}} \text { combination band of alcohols] }\end{array}$ \\
\hline $\begin{array}{l}2150-2200\{4651- \\
4545\}\end{array}$ & 6 & Combination $\nu_{C H}+[$ characteristic of (cis) internal instauration $]$ \\
\hline $2240\{4464\}$ & 7 & Combination $\nu_{C H}+$ [characteristic of $\mathrm{CH}_{3}-$ groups $]$ \\
\hline $\begin{array}{l}2300-2480\{4347- \\
4032\}\end{array}$ & 7 and 8 & Combination $\nu_{\mathrm{CH}}+\left[\right.$ characteristic of $-\mathrm{CH}_{2}-$ groups $]$ \\
\hline
\end{tabular}

$* \nu_{C H}$ stands for all possible combination modes of $\mathrm{CH}$ bonds, including symmetric and asymmetric vibration, stretching, rotation, torsion. 


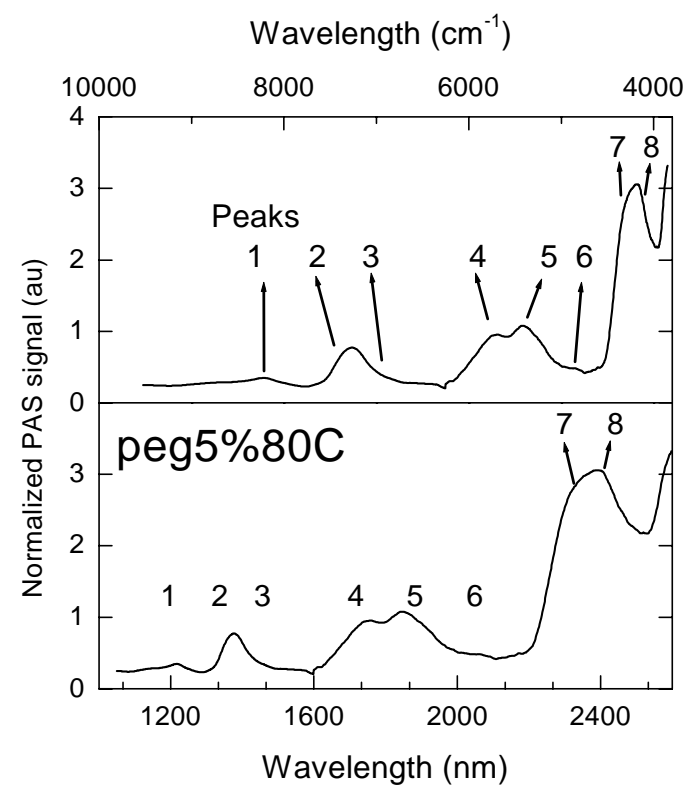

Figure 6. PA spectrum of a cross-linked polymer in the IR range. Grafted PE with $5 \%$ catalyst under water vapor at $80{ }^{\circ} \mathrm{C}$.

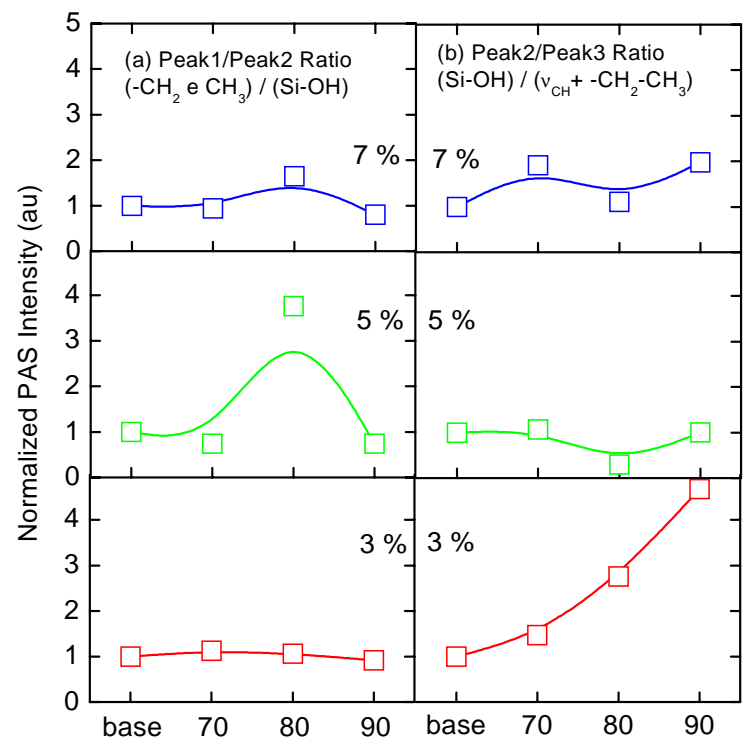

Figure 7. PA intensity ratios for $\mathrm{PEg}$ in NIR range (Cop presented the same result).

The PA spectra in the MIR region are shown in Fig. 8 and this is another useful example given for samples Cop770 and PEg770, respectively. For example, Fig. 9 shows only the phase separation of the Cop770, where peaks 4 and 5 are well defined and separable. In contrast, peaks 7 and 8 are very difficult to be visually separated in this figure. In fact, we found no phase difference in between peaks 7 and 8 for both samples, Cop770 and $\operatorname{PEg} 770\left(\Delta \phi \sim 0^{0}\right)$ as it is shown by the two lower frames of Fig. 9. On the other hand, it was found $\Delta \phi \sim 24^{0}$ for Cop770 (Fig. 9) and $\Delta \phi \sim 27^{0}$ for PEg770 (figure not shown).

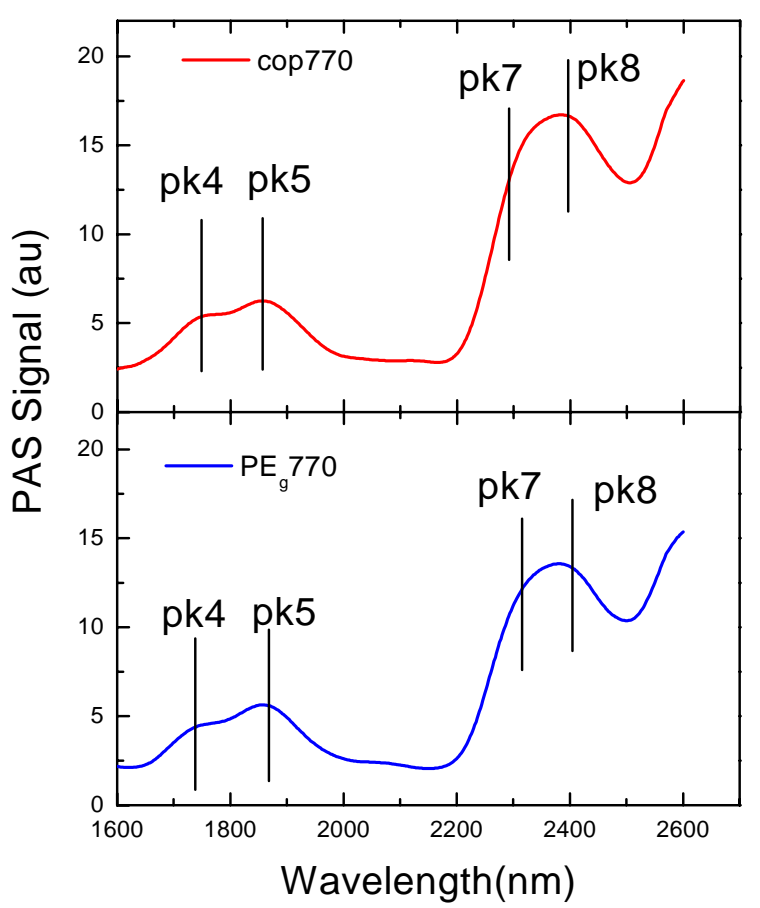

Figure 8. Spectral signal for a cross-linked polymer at $7 \%$ catalyst and $70{ }^{\circ} \mathrm{C}$ in MIR range.

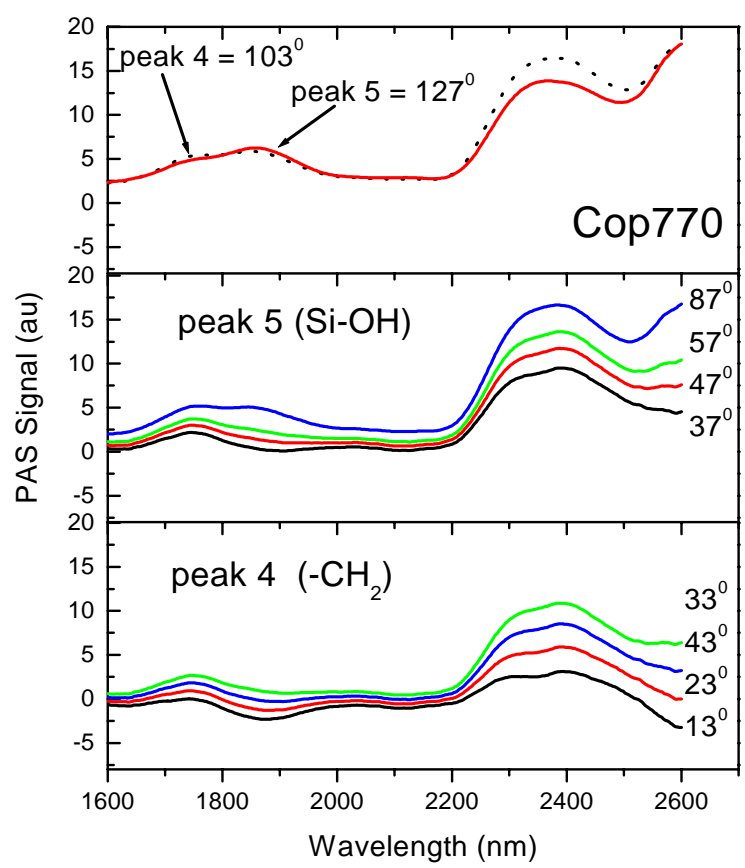

Figure 9. Examples of numerical phase separation for peaks 4 and 5 (resolved) and for peaks 7 and 8 (not resolved). Note that peaks 7 and 8 are simultaneous (no phase difference).

Making the numerical analysis, we are able to separate the contribution of free $O H$ and the $1^{\text {st }}$ overtone 
of the methylene $-\mathrm{CH}_{2}$. As expected, the PA analysis showed no separation for the stretching combination $v_{\mathrm{CH}}$ that is attributed to $-\mathrm{CH}_{2}$ and $-\mathrm{CH}_{3}$ overtones. This observation is exemplified by Fig. 9 whilst the overall phase separations of these samples are shown in the phasorial diagram, in Fig. 10a, for PEg770, and in Fig. 10b, for Cop770.

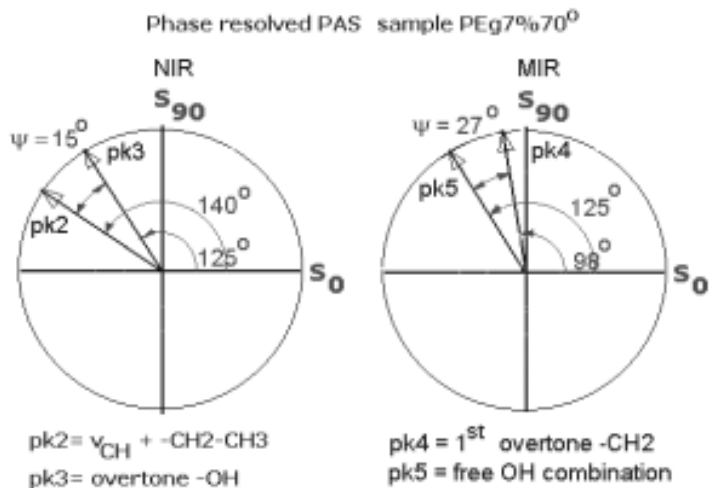

(a)

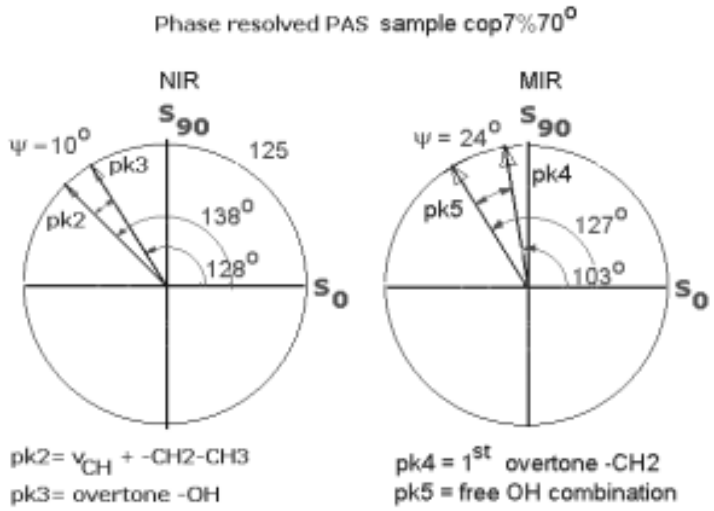

(b)

Figure 10. Phasorial diagram of separated components for both range NIR and MIR. a) For PEg770, and b) Cop770.

\section{Thermal diffusivity measure- ments}

\section{VI.1 Polymers foils and resins}

Many researchers in the material science field are interested in thermal parameters, specially in thermal diffusivity $(\alpha)$ measurements [44]. From $\alpha$ one can access the thermal conductivity $\kappa=\alpha \rho C_{p}$, also thermal diffusivity may reflect indirect effects in the solid lattice that are related to structural changes, like crystallinity, doping effects and processing conditions.

Thermal diffusivity based polymer foils studies have been reported by several papers $[27,26]$. For instance, studies such as drawn effects in PET [45],
Polyester with different liquid backing [46], different conducting foils like Polypyrrole (PPY) and Polyaniline $[47,48,49]$, temperature dependence of thermal parameters in Polyvinylidene (PVDF) films [50], color cellophane films [51], are found in literature. Also many others methods based on a two-layered polymer, such as Polymethyl Methacrylate (PMMA) on stainless steel and Mylar on glass plate [52, 53], or based on multilayered polymer [54, 55] are published. Particularly, they called attention to the great influence of thermal conductivity ratio on the effective thermal diffusivity of the two-layer system [53].

Phase Lag PA method and Thermoelastic bending have been exploited by Leite [27] and the OPC have been demonstrated by Perondi [26] for probing the technique for the measurement of thermal diffusivity of a set of polymer foils. Leite tabulated data for Teflon, Poly Vinyl Chloride (PVC), Cellulose Acetate (AA), Polypropylene (PP) and LDPE by using front phase data fitting. Perondi presented data for LDPE, HighDensity Polyethylene (HDPE) and PP, based on OPC measurements. Table II shows a little survey of properties for some polymers.

In addition, PA method allows the study of crosslinking of LDPE and some Epoxy Resins. Cella et all [56] have studied the LDPE after being swollen into a Dicumyl Peroxide (Di-Cup) but the cross-linking process was followed by means of thermal diffusivity showing that this parameter increases with immersion time of the order of 10 hours, saturating for time up to 30 h.ours Also d'Almeida et al. [57, 58] showed that thermal diffusivity is very sensitive for monitoring the changes in epoxy-amine, induced on the macromolecular network as a function of hardener/resin ratio. Extending this work, d'Almeida reported that thermal diffusivity is suitable to monitor the fracture behavior of the epoxy resin under impact conditions, when different hardeners are used.

\section{VI.2 Modified and Dyed Polyethylene Tereph- thalate}

The PA spectroscopy has been used to monitor the processing variable in dyeing commercial PET films modified by DMMA [59]. The net effects in the microstructure were accompanied using the OPC PA cell, in which thermal diffusivity were measured for a set of sample, with different dyeing conditions (dyeing time and temperature) [60]. In the study presented by Olenka et al. $[60,61]$ it was used a $100 \mu \mathrm{m}$ thick foils of PET films. The main results have pointed that the PET film improved the heat conduction power after dyed at temperature below glass transition $\left(T<70^{\circ} \mathrm{C}\right)$ when time of dyeing is kept at $30 \mathrm{~min}$. On the other hand, for the set of sample dyed at higher temperature $\left(T>70^{\circ} \mathrm{C}\right)$ the results showed that the thermal diffusivity decreases drastically for a 6 hours swelling time. 
Table II - Tabulated physical properties of some polymers retrieved using PA.

\begin{tabular}{|c|c|c|}
\hline Polymer & $\begin{array}{l}\text { Physical Properties, } \\
\alpha\left(10^{-3} \mathrm{~cm}^{2} / \mathrm{s}\right)\end{array}$ & Reference \\
\hline $\begin{array}{l}\text { 1-Red Polyester } \\
\text { on transparent PS }\end{array}$ & $\begin{array}{c}1.3\left(\beta>800 \mathrm{~cm}^{-1}\right) \\
\left(\sim 10 \mathrm{~cm}^{-1}\right)\end{array}$ & Double Layer Model[55] \\
\hline 2-PETP drawn foils & & Rear phase dependence[45] \\
\hline$l s^{\sim} 19 \mu \mathrm{m}$ to $18 \mu \mathrm{m}$ & $0.92==>0.75$ & \\
\hline$l s^{\sim} 30 \mu \mathrm{m}$ to $31 \mu \mathrm{m}$ & $0.62==>0.67$ & \\
\hline $\begin{array}{l}3 \text {-Mylar/Glass e } \\
\text { depends on } k_{1} / k_{2}\end{array}$ & $0.45<\alpha_{e f f}<0.82$ & Two Layer System Model[53] \\
\hline 4-Polyester & 0.9 & Backing liquid Method[46] \\
\hline 5-Teflon & 1.4 & Phase Lag and Bending Method [27] \\
\hline PVC & 0.6 & \\
\hline $\mathrm{AA}$ & 0.8 & \\
\hline PP & 0.7 & \\
\hline LDPE & 1.6 & \\
\hline 6-HDPE & 1.7 & OPC - Rear phase dependence[26] \\
\hline 7-PET & 1.02 & OPC - Rear signal dependence[61] \\
\hline DMMA treated & $1.2<\alpha<1.3$ & \\
\hline Blue Disperse Dyed & $1.4<\alpha<2.2$ & \\
\hline $\begin{array}{l}\text { 8-PMMA red film } \\
\text { under electric field }\end{array}$ & 0.6 & OPC configuration [62] \\
\hline $\begin{array}{l}\text { 9-Iodine doped PST } \\
\text { doping time }(\mathrm{min})\end{array}$ & $\begin{array}{c}\tau \sim 20 \mathrm{~ms} \\
\tau_{\min }(180) \sim 10 \mathrm{~ms}\end{array}$ & OPC - Rear phase dependence[41] \\
\hline & $\tau_{\beta \text { average }} \sim 60 \mathrm{~ms}$ & \\
\hline & $\tau_{\beta \text { peak }}(180) \sim 80 \mathrm{~ms}$ & \\
\hline
\end{tabular}

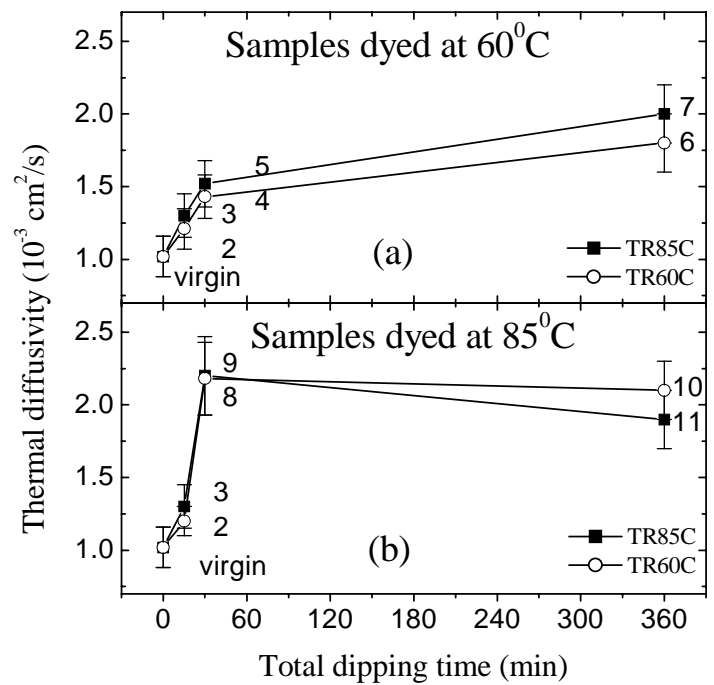

Figure 11. Thermal diffusivity results for treated PET as a function of time of dyeing. (a) Samples dyed at $60{ }^{\circ} \mathrm{C}$ and treated at both $60{ }^{\circ} \mathrm{C}$ and $85^{\circ} \mathrm{C}$ and; (b) Samples dyed at $85^{\circ} \mathrm{C}$ and treated at both $60{ }^{\circ} \mathrm{C}$ and $85^{\circ} \mathrm{C}$. Lines are guides to the eyes.

Thermal diffusivities are plotted in Fig. 11 against dyeing time. Thermal diffusivity increases higher for the set dyed above PET glass transition, frame (b), showing that it decreases for long term dyeing ( 6 hours typically). In contrast, for the set dyed at temperature below glass transition, frame (b), it presented a different behavior where thermal diffusivity increases all the way as time goes, but even so it is not linear. A summary of these results is presented in Table III.

The calculated thermal conductivity is plotted against integrated x-ray intensity area in Fig. 12. It shows a linear behavior for samples treated at $60^{\circ} \mathrm{C}$. In contrast, samples treated at $85^{\circ} \mathrm{C}$ showed an errant behavior that is suggested as a micro-structural changes taking place in the lattice.

\section{Final Remarks}

PAS have been shown to be a very useful optical technique for studying optical and thermal properties of polymers. Particularly when depth profile analysis is needed, the frequency domain allows one to study properties of layered sample and separate layer contribution. A promising method is the phase resolved PAS that can give us insight about superimposed absorption bands in the NIR and MIR region that is very rich in overtone and combinations of vibrational modes, usually found in most polymer, copolymer, blends and resins. Finally, PA method is very helpful for thermal parameters achievement, and the Phase Lag methods and the OPC have proven to be worthy for deriving thermal diffusivity for flat and transparent polymer, such as slabs or foils. 
Table III - Results of physical properties for each PET treated 15 min with DMMA and dyebath concentration 2 $\% \mathrm{Mol} . \mathrm{L}^{-1} . \mathrm{TR}=$ temperature of treatment, DY=temperature of dyeing, DT=time of dyeing.

\begin{tabular}{|c|c|c|c|c|c|c|}
\hline Sample & & $\bar{b}$ & $\begin{array}{c}\overline{\overline{\alpha_{s}}} \\
\left(10^{-3} \mathrm{~cm}^{2} / \mathrm{s}\right)\end{array}$ & $\begin{array}{c}\rho \\
\left(g / \mathrm{cm}^{3}\right) \\
\pm 0.005\end{array}$ & $\begin{array}{c}c_{p} \\
(J / g K) \\
\pm 0.04\end{array}$ & $\begin{array}{c}k_{s} \\
(m W / c m K) \\
\pm 0.6\end{array}$ \\
\hline 1 & Virgin PET & -0.554 & $1.02 \pm 0.14$ & 1.386 & 1.32 & 1.9 \\
\hline 2 & TR60 & -0.510 & $1.21 \pm 0.14$ & 1.345 & 1.24 & 2.0 \\
\hline 3 & TR85 & -0.492 & $1.30 \pm 0.15$ & 1.357 & 1.23 & 2.2 \\
\hline 4 & TR60DY60DT30 & -0.468 & $1.43 \pm 0.15$ & 1.368 & 1.23 & 2.4 \\
\hline 5 & TR85DY60DT30 & -0.455 & $1.52 \pm 0.16$ & 1.368 & 1.27 & 2.6 \\
\hline 6 & TR60DY60DT6 & -0.415 & $1.82 \pm 0.19$ & 1.368 & 1.32 & 3.3 \\
\hline 7 & TR85DY60DT6 & -0.395 & $2.02 \pm 0.21$ & 1.387 & 1.26 & 3.5 \\
\hline 8 & TR60DY85DT30 & -0.379 & $2.18 \pm 0.25$ & 1.368 & 1.20 & 3.6 \\
\hline 9 & TR85DY85DT30 & -0.378 & $2.20 \pm 0.27$ & 1.368 & 1.21 & 3.6 \\
\hline 10 & TR60DY85DT6 & -0.382 & $2.14 \pm 0.14$ & 1.368 & 1.20 & 3.5 \\
\hline 11 & TR85DY85DT6 & -0.411 & $1.86 \pm 0.19$ & 1.357 & 1.19 & 3.0 \\
\hline
\end{tabular}

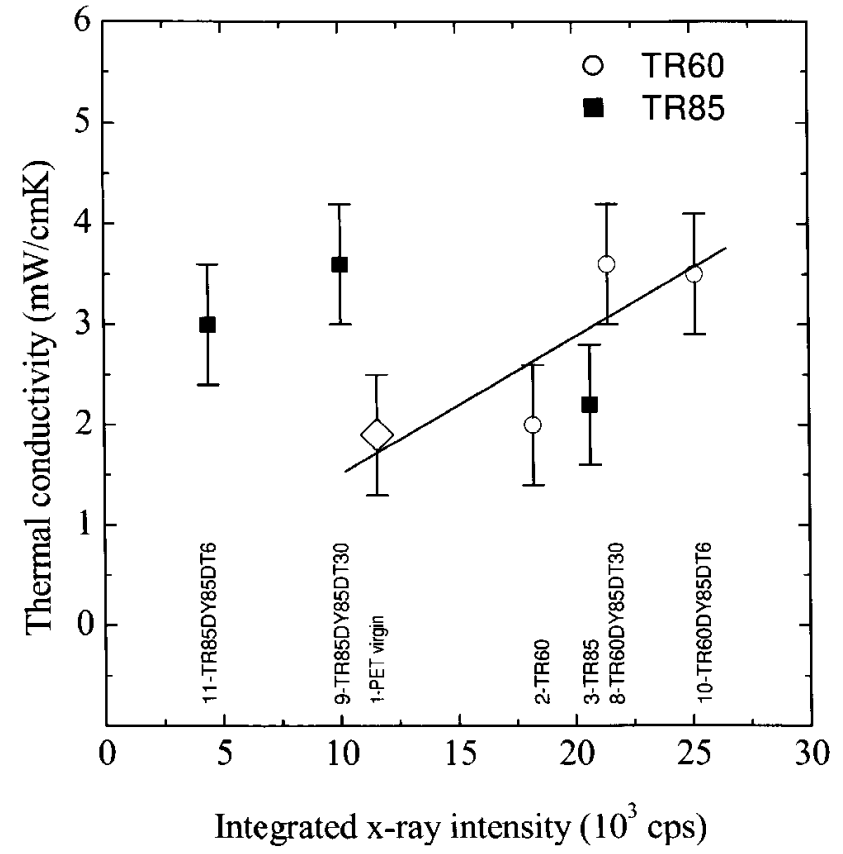

Figure 12. Calculated thermal conductivity for dyed PET films as a function of x-ray integrated peak area. The line points to the tendency of increasing the thermal conductivity with crystallinity.

\section{Acknowledgments}

We are in debt with the Brazilian Agencies Capes, $\mathrm{CNPq}, \mathrm{PADCT}$ and Fundação Araucária for the financial support of our research group.

\section{References}

[1] A.G. Bell, Am. J. Sci. 20, 305 (1880).
[2] A.C. Boccara, D. Fournier, and J. Badoz, Appl. Phys. Lett. 36, 130 (1980).

[3] A. Mandelis, Chem. Phys. Lett. 108, 388 (1984).

[4] H. Coufal, Appl. Phys. Lett. 44, 59 (1984).

[5] W. Jackson and N.M. Amer, J. Appl. Phys. 51, 3343 (1980).

[6] J. Mura, L.C.M. Miranda, M.L. Baesso, A.C. Bento, and A.F. Rubira, J. Appl. Pol. Sci. 82, 2669 (2001).

[7] S.O. Kanstad, P.E. Nordal, Powder Technol. 22, 133 (1978).

[8] A. Rosencwaig and A. Gersho, J. Appl. Phys. 47, 64 (1976).

[9] M.D. da Silva, I.N. Bandeira, and L.C.M. Miranda, J. Phys.E:Sci. Instrum. 20, 1476 (1987).

[10] A. Rosencwaig, Photoacoustic and Photoacoustic Spectroscopy, Wiley, New York, 1980.

[11] C.K.N. Patel and A.C. Tam, Rev. Mod. Phys. 53, 517 (1981).

[12] K. Klein, J. Pelzl, and H. Futterer, Photoacoustic, Principles and Applications, eds. E. Lüsher, P. Korpiun, H. J. Coufal and R. Tilgner, Viewg, Braunschweig, 1982.

[13] H. Vargas and L.C.M. Miranda, Photoacoustic and Related Photothermal Techniques; Phys. Rep. 161, 43 (1988).

[14] E. Helwig, B. Sandner, U. Gropp, F. Vogt, S. Wartwig, and S. Henning, Biomaterials 22, 2695 (2001).

[15] L. Gonon, J. Mallegol, S. Commereuc, and V. Verney , Vibrat. Spectr. 26, 43 (2001).

[16] M.B. Hocking, K.A. Klimchuk, and S. Lowen, J. Pol. Sci. A - Pol. Chem. 39, 1960 (2001).

[17] P. Schmidt, J. Baldrian, J. Scudla, and J. Dybal, M. Raab, K.J. Eichhorn, Polymer, 42, 5325 (2001).

[18] R.A. Palmer, Microbeam Analysis, Proc. 165, 47 (2000). 
[19] M.W. Urban, Progr. in Organ. Coatings, 40, 195 (2000).

[20] F.A. McDonald, J. Opt. Soc. Am. 70, 555 (1980).

[21] P. Charpentier, F. Lepoutre, and L. Bertrand, J. Appl. Phys. 53, 608 (1982).

[22] G. Rousset, F. Lepoutre, and L. Bertrand, J. Appl. Phys. 54, 2383 (1983).

[23] P. Korpiun and B. Büchner, Appl. Phys. B30, 121(1983)

[24] A.C. Bento, Master Dissertation; presented to IFGWUNICAMP, Campinas-SP, Brazil, 1987.

[25] G. M. Sessler, J. Acoust. Soc. Am. 35, 1354 (1963).

[26] L.F. Perondi and L.C.M. Miranda, J. Appl. Phys. 62, 2955 (1987).

[27] N.F. Leite, N. Cella, H. Vargas, and L.C.M. Miranda, J. Appl. Phys. 61, 3025 (1987).

[28] C.L. Cesar, H. Vargas, J. Pelzl, and L.C.M. Miranda, J. Appl. Phys. 55, 3460 (1984).

[29] M.L. Baesso, Doctoral Thesis presented to IFGWUNICAMP, Campinas-SP, Brazil, 1990.

[30] F.A. McDonald, G.C. Wetsel Jr., J. Appl. Phys. 49, 2313 (1978)

[31] O. Pessoa Jr., C.L. Cesar, N.A. Patel, H. Vargas, C.C. Chizoni, and L.C.M. Miranda, J. Appl. Phys. 59, 1316 (1986).

[32] S. Kammer, K. Albinsky, B. Sandner, and S. Wartewig, Polymer 40, 1131 (1999).

[33] J.M. Stegge, M.W. Urban, Polymer 42, 5479 (2001).

[34] B.R. Kiland, M.W. Urban, and R.A. Ryntz, Polymer 42, 337 (2001).

[35] H. Kim, M.W. Urban, Langmuir 16, 5382 (2000).

[36] B.R. Kiland, M.W. Urban, and R.A. Ryntz, Polymer 41, 1597 (2000).

[37] L. Gonon, O.J. Vasseur, and J.L. Gardette, Appl. Spectr. 53, 157 (1999).

[38] R.M. Dittmar, J.L. Chao, and R.M. Palmer, Appl. Spectr. 45, 1104 (1991).

[39] M.G. Oliveira, O. Pessoa Jr., H. Vargas, and F. Galembeck, J. Appl. Pol. Sci. 35, 1791 (1988).

[40] C.L. Cesar, C.A.S. Lima, N.F. Leite, H. Vargas, A.F. Rubira, and F. Galembeck, J. Appl. Phys. 57, 4431 (1985).

[41] A. Torres-Filho, N.F. Leite, L.C.M. Miranda, N. Cella, and H. Vargas, J. Appl. Phys. 66, 97 (1989).

[42] L. Olenka, É. N. da Silva, W.L.F. dosSantos, A.F. Rubira, E.C. Muniz, A.N. Medina, M. L. Baesso, and A.C. Bento, The Analyst, 127, 300 (2002).
[43] D.T. Dias, Master Dissertation presented to DFIUEM, Maringá-PR, Brazil, 2001. D.T. Dias, M.F. Porto, A. F. Rubira, A.N. Medina, M.L. Baesso, and A.C. Bento, submitted to J. Appl. Pol. Sci. 2002.

[44] L.R. Touloukian, R.W. Powell, C.Y. Ho, and M. C. Nicolasu, Thermal Diffusivity, vol. 10, IFI/PLENUM, New York(1973).

[45] P. Korpiun, B. Merté, G. Fritsch, R. Tilgner, and E. Lüscher, Coll.\& Pol. Sci. 261, 312 (1983).

[46] A. Lachaine and P. Poulet, Appl. Phys. Lett. 45, 953 (1984).

[47] A.C.R. da Costa and A.F. Siqueira, J. Appl. Phys. 80, 5579 (1996).

[48] W.L.B. Mello and R.M. Faria, Appl. Phys. Lett. 67, 3892 (1996).

[49] J.E. de Albuquerque, W.L.B. Mello, and R.M. Faria, J. Appl. Pol. Sci. B-Pol. Phys. 38, 1294 (2000).

[50] B. Bonno, J. L. Laporte, and R. T. d 'Leon, Meas. Sci. Technol. 12, 671 (2001).

[51] A. Yoshida, H. Nogami, T. Kurita, and S. Washio, Annal. Sci. 17, s154 (2001).

[52] T. Tominaga, K. Ito, Jap. J. Appl. Phys. 27, 2392 (1988).

[53] A.M. Mansanares, A.C. Bento, H. Vargas, N.F. Leite, and L.C.M. Miranda, Phys. Rev. B 42, 4477 (1990).

[54] P. Helander and I. Lundström, J. Appl. Phys. 52, 1146 (1981).

[55] M. Morita, Jap. J. Appl. Phys. 20, 835 (1981).

[56] N. Cella, H. Vargas, E. Galembeck, F. Galembeck, and L.C.M. Miranda, J. Appl. Pol. Sci.-C, 27, 313 (1989).

[57] J.R.M. d 'Almeida, N. Cella, S.N. Monteiro, and L.C.M. Miranda, J. Appl. Pol. Sci. 69, 1335 (1998).

[58] J.R.M. d 'Almeida and N. Cella, J. Appl. Pol. Sci. 77, 2486 (2000).

[59] W.L.F. dos Santos, M.F. Porto, E.C.Muniz, L. Olenka, M.L. Baesso, A.C. Bento, and A.F. Rubira, J. Appl. Pol. Sci. 77, 289 (2000).

[60] L. Olenka, É. N. da Silva, W.L.F. dos Santos, A.F. Rubira, E.C. Muniz, A.N. Medina, L.P. Cardoso, M.L. Baesso, L.C.M. Miranda, and A.C. Bento, Annal. Sci. 17, s387 (2001).

[61] L. Olenka, É.N. da Silva, W.L.F. dos Santos, E.C. Muniz, A.F. Rubira, A.N. Medina, L.P. Cardoso, L.C.M. Miranda, M.L. Baesso, and A.C. Bento, J. Phys. D: Appl. Phys. 34, 2248 (2001).

[62] H. Kobayachi, K. Yoshida, M. Kubo, T. Tsukada, M. Hosawa, and M. Sato, J. Chem. Eng. Japan 33, 47 (2000). 